\title{
An Agent-Based Social Simulation Platform with 3D Representation for Labor Integration of Disabled People
}

\author{
Alberto Barriuso ${ }^{1}$, Fernando De la Prieta ${ }^{1}$, and Tiancheng $\mathrm{Li}^{2}$ \\ ${ }^{1}$ Department of Computer Science and Automation Control. University of Salamanca, \\ Plaza de los caidos s/n, 37077, Salamanca \\ \{albarriuso, fer\} @usal.es \\ ${ }^{2}$ School of Mechanical Engineering. Northwestern Polytechnical University, \\ Xi' an, 710072, P.R. China \\ t.c.li@mail.nwpu.edu.cn
}

\begin{abstract}
In this paper, we present the design and implementation of a novel agent-based platform with 3D representations for labor integration. In order to evaluate and validate the proposed platform, a case study in a real workplace environment is modeled, with the purpose of identifying architectural barriers that hinder the accessibility and the processes of the workplace environment, allowing us to improve the accessibility of the environment to disabled people. Thereby, through the incorporation of novel simulation technics and mechanisms, it is possible to find areas that may be improved.
\end{abstract}

Keywords: Multi-agent system, agent-based simulation, 3D representation, jade.

\section{$1 \quad$ Introduction}

According to studies carried out by the United Nations (UN) [26], around $15 \%$ of the world population suffers some kind of disability, either physical or psychological, which hinders or impede them to carry out their regular daily routines in all the scopes (work, education, personal, etc.). Inasmuch as this collective faces clear disadvantages in their dairy routines to a greater or lesser extent, it is necessary to undertake all possible efforts to minimize these difficulties.

The integration of disabled people among the working environment is one of the mayor challenges in this regard, since it does not just allow the self-sufficiency of disabled people, but it also facilitates their social integration, and their self-esteem improvement. However, this is a large challenge. Speaking in quantitative terms, the International Labour Organization (ILO) points that there are around 386 million working-age people who suffer some kind of disability. This rate reaches values around the $80 \%$ in some countries [17].

There is no doubt that the current society is concerned about this challenge. In addition, there is a growing preoccupation from the different governments, public organizations and private entities. In this regard, since the declaration of the Standard Rules 
on the Equalization of Opportunities for Persons with Disabilities by the UN [25], different national, regional and local governmental organizations have developed specific regulatory frameworks that have improved the everyday lives of people with disabilities, where we can highlight, at European level, the Charter of Fundamental Rights of the European Union [4], the Treaty on the Functioning of the European Union [7], the Council Directive 2000/78/EC [14] and the European Disability Strategy 2010-2020 [6]. These initiatives go in hand with a growing academic attention, which has allowed the development of systems, techniques, models and methodologies oriented to improve the accessibility [18]. So, as example, we can highlight the work proposed by [9] that presents a software user-friendly man machine interface for accessing AT software in cloud computing or [20], where an obstacle avoidance system and a Fuzzy Logic Controller (FLC) adapted to a wheelchair is presented.

Beyond the development of these artifacts, one of the most promising study fields is social simulation, which in general terms, is applied to obtain a higher knowledge of a certain phenomenon which is being studied [5]. In the framework of the disabled people occupational integration, simulations can allow discovering in advance the problems which workers with disabilities will find before this problems occur. Within the scope of this research work, a new multi-agent system (MAS) based simulation platform is proposed. This platform makes use of 3D models of the workplace in order to perform accurate simulations, allowing at the same time to collect relevant and high quality data. Through the use of the proposed platform, it is possible to investigate in new mechanisms oriented to the decision making regarding the workflows present in the environment, the architectonical barriers detection, etc. This will allow the improvement of the workplace environment accessibility.

The rest of this paper is organized as follows: section 2 provides a brief introduction to the Agent-Based Social Simulation (ABSS) theory, Section 3 defines the proposed system, Section 4 evaluates the proposed system through a case of study and we conclude and introduce future research topics in Section 5.

\section{$2 \quad$ Agent Based Social Simulation}

ABSS is one of the most representative techniques which are used to complex inquiries where a large number of active and heterogeneous objects are present. These objects can be humans [21], business units [16], functional or nonfunctional objects [27], animals [23], etc. The phenomenon to be simulated is a set of sequences or events in a system (natural or artificial), which can exist (or not) in the real (or artificial) world and can be configured in the simulation model. These tasks are interrelated, since they finally are time or order dependent within the events which take place in the system. In this way, the simulation model allows the implementation of different and specific functionalities for each kind of target, applying different degrees of freedom. The complexity lies in the fact that this kind of models allow the simulation of complex and changing events, so the use of intelligent agent based techniques is appropriate, arising in this direction the ABSS concept [10]. This technique is focused on the social phenomenon simulation, using MAS models. Therefore ABSS is a combination of social sciences, agent-based computation and computational simulation. 
The use of this techniques is specially indicated when it is necessary to capture different tasks, elements, objects, persons in dynamic complex environments, as long as they can be implemented without having a deep knowledge about the global interdependencies. Moreover, it also allows facilities on changing models, since it is not necessary to make local changes, but global [2]. Besides, the benefits of agent based computing for computer simulation include various methods for evaluating MAS or for training future users of the system [11].

The use of this models and tools in ABSS allows modeling a great variety of tasks and environments. In fact, it has been applied in previous works, obtaining good results [13], [3]. However, it has been observed that there are certain difficulties when modeling the physical environment which we intend to simulate [19]. This feature is especially relevant when modeling simulations oriented to the disabled people workplace integration, since a high problem percentage of the problems to be solved are related with the structure of buildings, or accessibility barriers (steps, ramps, lifters, doors, corridors, etc.).

\section{$3 \quad$ Agent-Based Platform for Simulating Working Environments}

In this section the proposed platform is presented. One of the main aims to be achieved with its design and development has been the capability of modeling different environments in a flexible and dynamic way. For that reason, a novel combination of virtual organization (OV) based MAS has been used [15], allowing to model the human organization and a $3 \mathrm{D}$ environment representation where simulations can be carried out. A representation of the proposed simulation architecture is illustrated in Fig. 1.

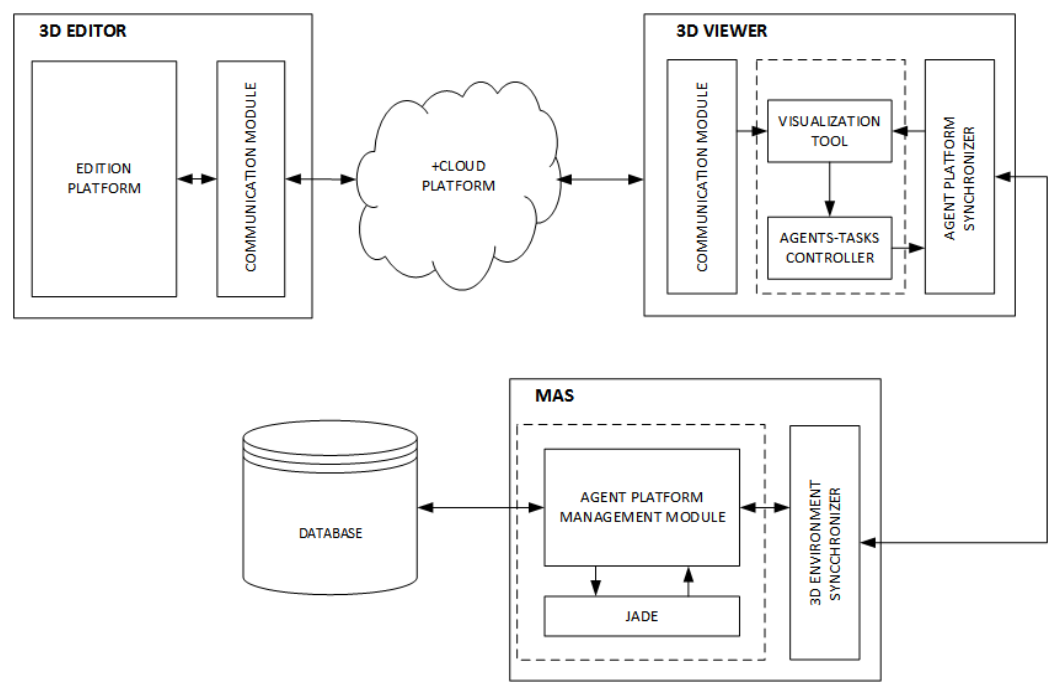

Fig. 1. Agent-based simulation platform 
The combination of both strategies is a key factor to achieve the main objective which we pretend to simulate. On one hand, agents can implement different tasks, objectives, purposes, etc.; and in the other hand, the 3D environment allows modeling physically the workplace environments. Thereby, this approach allows carrying out simulations using the workplace environment itself, as the visualization of their results.

\subsection{Components of the Proposed Architecture}

On the one hand, MAS consists of intelligent agents which represent and allows to simulate the desired model. The MAS is based on VO [24], allowing the representation of a structure, roles, as well as a wide range of rules which will schedule the interactions among agents.

The MAS has been developed by means of JADE agent platform [1]. In this respect, JADE does not allow the implementation of this capabilities, so it has been necessary to include a higher layer, which is responsible for the MAS management from an organizational point of view. This layer, allows us to provide additional self-organizational capabilities to the platform, including: (i) the capacity of grouping the MAS as a VO, enabling the agents to adopt different topologies [12]; (ii) definition of the tasks attending to the role which the agent assumes inside the organization, as well as the definition of unique characteristics to each agent;(iii) the ability to define a set of rules to regulate the interactions among agents has been implemented. Furthermore, this software layer which is included over JADE also allows to define scheduling and task-allocating oriented agents within the simulation. Finally, this layer also allows the communication within the $3 \mathrm{D}$ environment, so it can notify all the changes that take place bidirectionally between the agent platform and the tridimensional environment.

Fig. 2 shows in detail the structure of each one of the agents, consisting of three main components: (i) the communication module, which will allow the agents to communicate with the other agents in the platform, (ii) a reasoning module, which is based on the BDI model [22] and (iii) the communication module between the agent and the 3D environment. This module is in turn made up of a sensor and an effector. It is necessary that these two elements are distributed, being one component present in the implementation of the agent itself (as a part of the agent platform) and the other one in the 3D environment. With this distribution of both the sensor and the effector, we allow the agent to be conscious about the tridimensional environment state which has been rendered for the current simulation, so the agent is able to monitor the changes which take place in the virtual world.

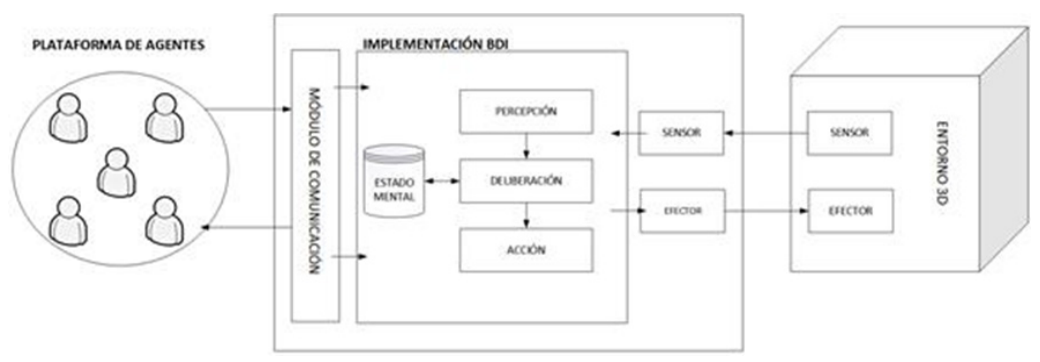

Fig. 2. Agent structure overview 
On the other hand, the 3D environment consists of an editor and a visualizer, both implemented using the Unity 3D engine -http://unity3d.com/-. Firstly, the 3D edition environment allows to model virtual tridimensional environments of urban areas (buildings, flats, apartments, gardens, parks, etc.) with a high level of quality and realism. All the maps and 3D models are stored remotely in a Cloud Computing (CC) platform. Secondly, the 3D visualizer makes possible the representation and rendering of both the environment and the characteristics that we have defined previously on the $3 \mathrm{D}$ editor, which we will get from the $\mathrm{CC}$ platform. It will also be responsible for displaying the agents which are part of the system, enabling the visualization of how each agent performs different tasks.

One of the main problems that we can find on the development of 3D scenes can be found when a model needs to move from one point to another one. In such cases, it is necessary to use an algorithm responsible for the path finding. On this occasion we make use of CritterAI [8], a navigation tool for use with Unity. It includes navigation mesh generation, pathfinding and local steering features, including implementations of both $\mathrm{A}^{*}$ and Dijsktra search algorithms.

\subsection{Communication between the Agents Platform and the Visualization Tool}

As we have highlighted previously, one of the main tasks in the platform is to establish communication between JADE and the 3D environment. On this way, we can offer a real corresponding between the events that take place at the platform of agents and the visualization, which occurs at the $3 \mathrm{D}$ platform. Inside the platform of agents, there will be an agent who is responsible of managing the communications from JADE.

With this purpose, communication between the platform of agents and the 3D environment will be done through TCP sockets, so with that purpose, it has been developed an Unity's module that manages the requests from the platform of agents and vice versa. On the side of JADE, an agent called Communication agent will be responsible of attending all the requests that will arrive from Unity and doing then the related tasks, attending to the exchanged frame.

Creation, elimination and interaction tasks between agents that are executed at the platform, must be updated at the 3D environment, and the creation, elimination and interaction tasks between agents that are requested from the $3 \mathrm{D}$ interface, must be carried out firstly at the platform of agents to be subsequently updated at Unity. The communication will be divided in two blocks:

- Sockets to simulate the JADE tasks and its corresponding update at Unity, with this purpose three elements are used: one socket to send the tasks, another one for confirming the tasks which have been carried out and one table for keeping a record.

- Sockets in charge of performing the tasks which are initialized from the Unity interface, which must be previously done in JADE to be later updated in Unity. 


\section{Case of Study}

The proposed simulation platform has been evaluated through a case of study, which is oriented to validate the proposed system. The scope of this case of study is the analysis of the accessibility level of different workstations in a real company, called Indra Software Labs, located in Salamanca, Spain. Through the performance of several simulations, we will be able to extract knowledge about how disabled workers can find different accessibility problems in the company during their usual activities.

In order to perform the simulations, it will be necessary to define the characteristics of the VO of agents, allowing to model de different processes which take place in the company as faithful to reality as possible. To that end, we will define all the different roles which agents can acquire, the necessary services for the correct performance of the organization, the norms which will govern the society and the messages and interactions between agents. In addition, we have to model the environment where a simulation takes place, in this case the building of the company under consideration. Subsequently, we will carry out a set of simulations in order to show how the organization behaves in different conditions, to finally effect a validation of the proposed model.

First, we define the interaction model, analyzing the needs of the system users, as well as the way in which the information exchange is carried out. De roles we define are as follows:

- Direction: this agent is responsible of planning the tasks which the whole organization will carry out. It generates the tasks which represent the objectives to be accomplished by the organization, delegating its distribution to the different agents in charge of the company areas, according to the nature of the task.

- Area/department responsible: an agent in charge of planning the distribution of the tasks which have been assigned to the department which it manages, attending to the availability and capabilities of the agents.

- Worker: agents that represent each one of the workers participating during the simulation. This kind of agent will have a stack where to store all the tasks that they have to carry out, and have been previously assigned through the area/department responsible. This agents will emit information about the degree of success which they have done each one of the tasks. All this agents share certain elements, which define a set of shared behaviors and cognitive capabilities between them. Beside of this shared characteristics, the agents will have their own characteristics, which are defined according its role in the organization, as well as its disabilities. The assigned roles will determine the tasks which an agent could do, as well as the specific behaviors for each one of them, enabling the possibility of modeling different executions of the same task, attending to the kind of disability that a worker might present.

- Environmental agents: represent those elements of the building which an agent can interact with in a direct way. We can find examples of environmental agents in elements such as telephones, lifters or photocopiers. 
Among the processes which take place in the company that we are studying, we have identified three kinds of departments, according to the tasks that workers perform: reception, administration and maintenance. The worker-kind agents will have associated a set of tasks that they can carry out, attending to the department which they belong to. Once the building model and the interaction model are defined, we will be able to perform the simulation. To that end, we shall deploy the visualization tool, which will need to perform certain operations before starting the simulation: (i) loading the building model, (ii) generating the navigation mesh, (iii) synchronization with the platform of agents. In Fig. 3 it is shown a visualization tool screenshot.

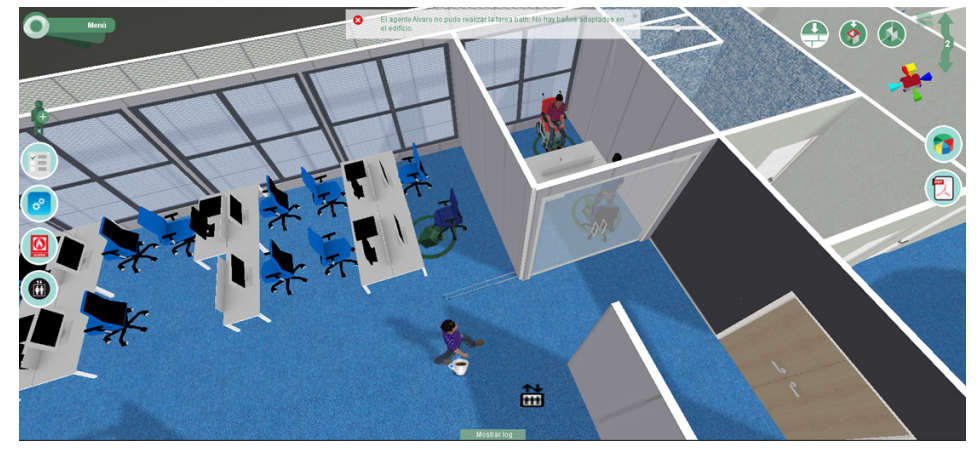

Fig. 3. 3D representation of the Social simulation

First there has been planned a 15 minutes simulation, with a total of 8 worker agents: 1 receptionist, 2 maintenance and 5 administration workers. Three of them present reduced mobility problems, three of them are visually impaired and one of them is deaf.

After the simulation, the tool generates a report which details all the accessibility problems which avatars have found, and have hampered the correct consecution of the tasks which were assigned to them. 118 tasks have been carried out correctly and 79 have failed (Fig. 4 (A)). Some of the limitations found by the system were: (i) lack of adapted bathrooms for disabled people in common areas, (ii) the workstations are not adapted to deaf or visually impaired people, making impossible to carry out tasks such as answering the phone, or being aware that the fire alarm is active, (iii) lack of access ramps to higher floors as an alternative to the lifter, which, in case of a breakdown will impede the access and exit to the building, (iv) limited access to certain workstations -spaces are not wide enough for a wheelchair- or (v) not accessible furniture elements -filing cabinet, photocopiers-.

Once this accessibility problems have been detected, a redistribution of the furniture elements whose accessibility may be improved, as well as the relocation of certain workstations of people using wheelchairs, or the adapting of certain workstations of people with visual or hearing impairment is proposed.

After this steps, we come to execute a new 15 minutes simulation, obtaining as result a total of 163 successful tasks and 28 failed tasks (Fig. 4 (B)), passing from a reduction of the $40,1 \%$ of the failed tasks in the initial simulation, to a $14,7 \%$ in the 
second simulation. Obviously, there are still some elements of the environment which will keep limiting its own accessibility, as the lack of access ramps or adapted bathrooms, but it has been proved that there is a possibility to improve the accessibility of the environment through the restructuration of the furniture or the workstations in the critical points which have been detected through the simulation.
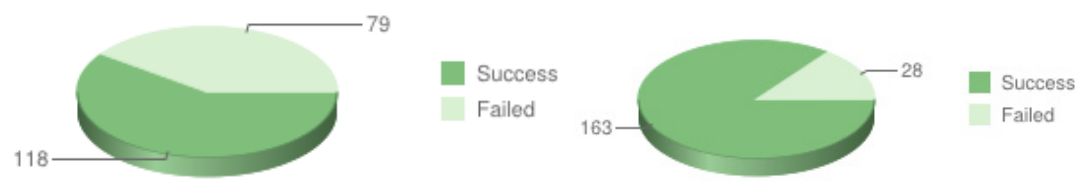

Fig. 4. A: First simulation results; B: Second simulation results

\section{Conclusions}

Once the simulation platform has been presented and evaluated, we can affirm that it allows performing simulations that are oriented to the integration of disabled people into the workplace, allowing the analysis of both the tasks which are done in the workplace and the environment physical characteristics where this tasks are performed. In this regard, a previous analysis about the state of art on related systems and models, as well as current legislation on this issue has been done.

Moreover, we have model a real organization through the use of a VO of agents, with self-adaptive capabilities. This system has been implemented using JADE, but adapting this platform to our special needs. In the same way, we have created a $3 \mathrm{D}$ environment, which is able to model and carry out simulations in the working environment. Additionally, we have developed a system which allows the communication between both platforms, enabling to share relevant information regarding the communications. In conclusion, this implementation as a whole allows simulating the processes which take place in a company, with the purpose of establishing which accessibility problems can occur among the working environment where the represented workers perform their work, as it has been shown on the case of study.

Acknowledgements. This work is supported by the Spanish government (MICINN) and European FEDER funds, project iHAS: Intelligent Social Computing for HumanAgent Societies (TIN2012-36586-C03-03).

\section{References}

1. Bellifemine, F., Poggi, A., Rimassa, G.: JADE-A FIPA-compliant agent framework. Proceedings of PAAM 99(97-108), 33 (1999)

2. Borshchev, A., Filippov, A.: From system dynamics and discrete event to practical agent based modeling: reasons, techniques, tools. In: Proceedings of the 22nd International Conference of the System Dynamics Society, vol. (22) (2004) 
3. Zato, C., Villarrubia, G., Sánchez, A., Barri, I., Rubión, E., Fernández, A., Rebate, C., Cabo, J.A., Álamos, T., Sanz, J., Seco, J., Bajo, J., Corchado, J.M.: PANGEA - platform for automatic coNstruction of orGanizations of intElligent agents. In: Omatu, S., Paz Santana, J.F., González, S.R., Molina, J.M., Bernardos, A.M., Rodríguez, J.M.C. (eds.) Distributed Computing and Artificial Intelligence. AISC, vol. 151, pp. 229-240. Springer, Heidelberg (2012)

4. Charter of Fundamental Rights of the European Union (2010/C 83/02)

5. Chung, C.A. (ed.): Simulation modeling handbook: a practical approach. CRC Press (2003)

6. Communication from the Commission to the European Parliament, the Council, the European Economic and Social Committee and the Committee of the Regions: European Disability Strategy 2010-2020: A Renewed Commitment to a Barrier-Free Europe (COM/2010/0636)

7. Consolidated version of the Treaty on European Union and the Treaty on the Functioning of the European Union 2012/C 326/01

8. CritterAi Documentation page, http://www.critterai.org/projects/ cainav/doc/(last access: January 27, 2015)

9. Mulfari, D., Celesti, A., Villari, M.: A computer system architecture providing a userfriendly man machine interface for accessing assistive technology in cloud computing. Journal of Systems and Software 100, 129-138 (2015) ISSN 0164-1212

10. Davidsson, P.: Agent based social simulation: A computer science view. Journal of Artificial Societies and Social Simulation 5(1) (2002)

11. Davidsson, P.: Multi agent based simulation: Beyond social simulation. In: Moss, S., Davidsson, P. (eds.) MABS 2000. LNCS (LNAI), vol. 1979, pp. 97-107. Springer, Heidelberg (2001)

12. Dignum, V.: Handbook of research on multi-agent systems: semantics and dynamics of organizational models, p. 111. Information Science Reference, Hershey (2009)

13. García, E., Rodríguez, S., Martín, B., Zato, C., Pérez, B.: MISIA: Middleware infrastructure to simulate intelligent agents. In: Abraham, A., Corchado, J.M., González, S.R., De Paz Santana, J.F. (eds.) International Symposium on Distributed Computing and Artificial Intelligence. AISC, vol. 91, pp. 107-116. Springer, Heidelberg (2011)

14. European Council Directive 2000/78/EC of 27 November 2000

15. de la Prieta, F., Pérez-Lancho, B., Francisco De Paz, J., Bajo, J., Corchado, J.M.: Ovamah: Multiagent-based adaptive virtual organizations. In: 12th International Conference on Information Fusion, FUSION 2009, pp. 990-997 (2009)

16. Prenkert, F., Følgesvold, A.: Relationship strength and network form: An agent-based simulation of interaction in a business network. Australasian Marketing Journal (AMJ) 22(1), 15-27 (2014) ISSN 1441-3582

17. International Labour Organization, Code of practice on managing disability in the workplace. Document TMEMDW/2001/2 (2001)

18. Bajo, J., Fraile, J.A., Pérez-Lancho, B., Corchado, J.M.: The THOMAS architecture in Home Care scenarios: A case study. Expert Systems with Applications 37(5), 3986-3999 (2010)

19. Corchado, J.M., Corchado, E.S., Aiken, J., Fyfe, C., Fer-nandez, F., Gonzalez, M.: Maximum likelihood Hebbian learning based retrieval method for CBR systems. In: Ashley, K.D., Bridge, D.G. (eds.) ICCBR 2003. LNCS, vol. 2689, pp. 107-121. Springer, Heidelberg (2003)

20. Masmoudi, M.S., Klabi, I., Masmoudi, M.: Fuzzy logic control and HMI interfaces on an intelligent wheelchair system. In: Proceedings-Copyright IPCO, pp. 253-260 (2014) 
21. Schreinemachers, P., Berger, T.: An agent-based simula-tion model of human-environment interactions in agricultural systems. Environmental Modelling \& Software 26(7), 845-859 (2011) ISSN 1364-8152

22. Rao, A.S., Georgeff, M.P.: BDI Agents: From Theory to Practice. In: ICMAS, vol. 95, pp. 312-319 (June 1995)

23. Almeida, S.J.D., Ferreira, R.P.M., Eiras, Á.E., Ober-mayr, R.P., Geier, M.: Multi-agent modeling and simulation of an Aedes aegypti mosquito population. Environmental Modelling \& Software 25(12) (December 2010)

24. Rodriguez, S., Julián, V., Bajo, J., Carrascosa, C., Botti, V., Corchado, J.M.: Agent-based virtual organization architecture. Engineering Applications of Artificial Intelligence 24(5), 895-910 (2011)

25. United Nations. Standard Rules on the Equalization of Opportunities for Persons with Disabilities (1994)

26. United Nations: Factsheet on persons with disabilities, http://www.un.org/ disabilities/default. asp?id=18 (last access: January 27, 2015)

27. Yu, Y., Kamel, A.E., Gong, G., Li, F.: Multi-agent based modeling and simulation of microscopic traffic in virtual reality system. Simulation Modelling Practice and Theory 45, 62-79 (2014) ISSN 1569-190X 